\title{
FREE SURFACE FLOW IN VERTICAL TAYLOR-COUETTE SYSTE
}

\section{TAKASHI WATANABE ${ }^{1}$ AND HIROYUKI FURUKAWA ${ }^{2}$}

\author{
${ }^{1}$ Graduate School of Informatics, Nagoya University \\ Furo, Chikusa, Nagoya 464-8601 Japan \\ watanabe@i.nagoya-u.ac.jp \\ ${ }^{2}$ Department of Mechanical Engineering, Meijo University \\ Shiogamaguchi, Tempaku, Nagoya 468-8502 Japan \\ furukawa@meijo-u.ac.jp
}

Key words: Free Surface, Vertical Cylinders, Taylor-Couette System, Mode Transition, Rotating Flow.

\begin{abstract}
Vertical Taylor-Couette flow with a free surface at its top has been examined by numerical and experimental approaches. Being compared with the ideal case with infinite length cylinders, this system has effects of the finite lengths, the gravitational acceleration and the surface tension. The inner cylinder rotates and the outer cylinder and the bottom wall are stationary. The numerical approach uses the VOF method to model the free surface. In the experiment, the flow patterns are observed from the top and the meridional views, and the displacement of the free surface is measured. The flow modes are classified by the number of vortices appearing in the meridional plane and the radial flow directions at the top and the bottom. The power spectra of the displacement of the free surface and the bulk energy are numerically evaluated and the bulk energy tends to give favorable agreement with the spectra of the displacement obtained experimentally. The transition from the secondary mode flow appearing at higher Reynolds number to the primary mode flow at lower Reynolds number is examined and comparison between the numerical and experimental results are made.
\end{abstract}

\section{INTRODUCTION}

Flows with free surfaces appear in fluid machinery, chemical reactors and ocean currents and they have scientific and practical interests. In this paper, we numerically and experimentally study the free surface flow between vertical rotating cylinders. Some characteristics of the free surface flow around a rotating cylinder were the targets of the study of viscous fingerings [1], [2]. After the pioneering and milestone study conducted about one hundred years ago [3], a series of modified flows with no main axial flow has been carried out on the flows between rotating cylinders with finite lengths [4].

The examination of air-liquid and liquid-liquid flow between rotating cylinders is interesting and important, and not a small number of studies have been performed. For the airliquid flows in horizontal cylinders, Hubacz et al. [5] and Dluska et al. [6] experimentally classified the flow patterns including stratified and slug flows and observed the interfacial 
area. The Peixinho et al. [7] measured the deformation of the free surface around a rotating inner cylinder in the flow at low liquid to air ratio and compared with the result of a numerical simulation considering the surface tension. They concluded that the free surface profile was well formulated by the capillary number. Baier et al. [8], Zhu et al. [9] and Nakase et al. [10] experimentally studied the two-liquids flow between horizontal cylinders. They examined the instability of the liquid-liquid interface and found the axially banded flow and radially separated flow. The mixture of water and kerosene flow in vertical rotating cylinders was studied by Sathe et al. [11]. They conducted the experimental and numerical study and compared the unsteady profile development of the interface and the dispersion process. Broadbent et al. [12] investigated the flow of the mixture of silicon oil and water in vertical rotating micro cylinders with free surface at the top and found the flow patterns similar to those in the horizontal cylinders [8]. However, they did not concern with the behavior of the free surface. Taylor-Couette system with a vertical axis and a free surface at the top has been studied in the case of the flows with or without an entrainment of the air. In the rotating flow with the entrainment, the bubbles were trapped near the boundaries of the vortices appearing in the meridional plane and the torque required to maintain the rotation would be reduced [13], [14]. Linek et al. [15] investigated the stability of flows with no entrainment. They determined the axial wave number at a given liquid height and referred to the Eckhaus boundary to investigate the stability. They also observed the reduction of the number of vortices in the decelerating flow. Deng et al. [16] estimated the profiles of the free surface by

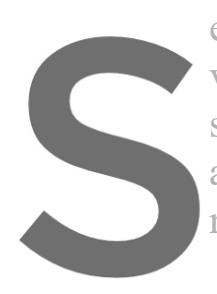
experimental observations and theoretical predictions and
velocity component when the flow became wavy. Vertical Tay
surface was also utilize to understand the rotating flow
azimuthally periodic flow with Rossby wave between the cy
radius ratio [18].

Taylor-Courette system with finite length
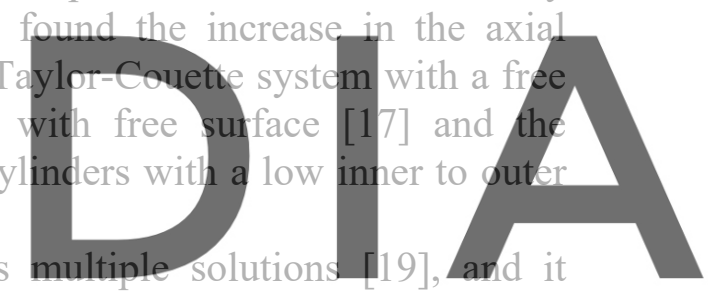

shows some flow modes. Let the inner cylinder rotate, while the outer cylinder is stationary.

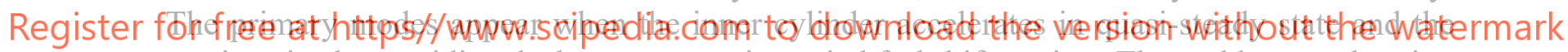
vortices in the meridional plane appear via a pitchfork bifurcation. The sudden acceleration and/or the higher Reynolds number make secondary modes with vortex numbers and flow directions different from those of the primary modes. Nakamura et al. [20] used the cylinders with both stationary ends and experimentally obtained the secondary mode flows. They also observed the transition processes of the well-developed secondary mode flows to the primary mode flows during the slow deceleration of the inner cylinder. The aspect ratio they adopted, which is defined by the fraction of the cylinder length to the gap width between two cylinders, is from 3.0 to 7.0. Watanabe et al. [21] numerically investigated the bifurcation result in [20] and obtained good agreement. Toya et al. [22] carried out their experimental study of the bifurcation between the secondary and primary modes in the vertical asymmetric TaylorCouette system with a top free surface and a stationary bottom wall. For their results, Watanabe et al. [23] simulated the system with an effect of surface tension at the small aspect ratio up to about 4.0 and evaluated the bifurcation diagram of the secondary and the primary modes. A new interest about the vertical Taylor-Couette system with free surface is the slant of the axis, and the effect of the tilt angle on the flow stability was examined [24], [25].

In this paper, we extend the study [23] and numerically investigate the vertical TaylorCouette system with a free surface at the top. We also conduct some experiments with our 
coworker who is one of the authors of study [22] and draw a comparison. Then, we investigate the developing process of the free surface flows, the flow modes, the unsteady behaviors of the well-developed flows and the transition from the secondary modes at higher Reynolds numbers to the primary modes.

\section{FORMULATION}

\subsection{Flows in Taylor-Couette system with vertical axis and free surface}

Taylor-Couette system considered in this paper consists of two vertical concentric cylinders. The inner cylinder with radius $r_{i}$ rotates and the outer cylinder with radius $r_{o}$ remains stationary. The bottom end of the cylinders is fixed wall. The lengths of the cylinders are long enough to let the working fluid have a free surface between the cylinders and the top and bottom conditions are asymmetric. The reference velocity is the circumferential velocity of the inner cylinder $U$ and the reference length is the cylinders' gap width $d=r_{0}-r_{i}$, and they define the Reynolds number $R e$. The height of the quiescent fluid is $L$ and the aspect ratio $\Gamma$ is given by $L / d$.

The flow mode is specified by the number of vortices and the flow directions at the top and the bottom in the meridional plane. The rotation of the inner cylinder brings a centrifugal effect. Consequently, the flow on the top free surface is radial outflow. On the other hand, the flow in a viscous Ekman layer on the botton is retarded and the flow direction is radially inward near the bottom normal flow, and then with inflow near the direction of the norma anomalous vortex.
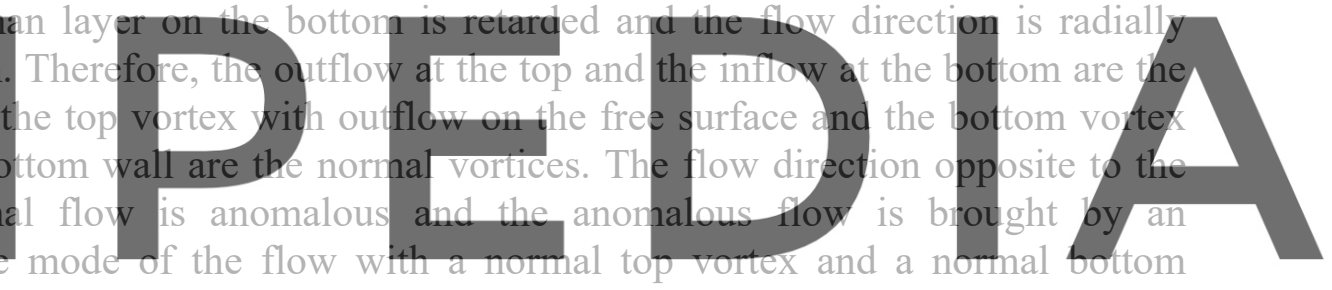
vortex is a normal mode. Otherwise, the flow mode is anomalous. When the normal mode

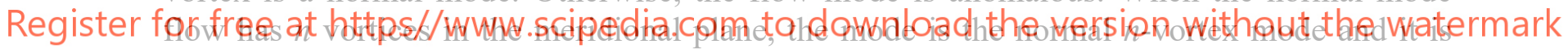
denoted by $\mathrm{N} n$. The anomalous mode flow with $n$ vortices is the anomalous $n$-vortex mode A $n$, and Aun means the mode of An with an upper top anomalous vortex.

Other than the normal and anomalous modes, the vortex flows are classified into the primary mode and the secondary mode. The primary mode represents the first vortex flow which appears after a quasi-steady acceleration of Couette flow. Even in the same geometrical flow field as that of the primary mode, the secondary mode flows have numbers of vortices and/or rotating directions different from those of the primary mode flow and they usually emerge at higher Reynolds numbers.

\subsection{Experimental and numerical methods}

The experimental setup by Toya [22] is used to measure and observe the flow. The radii of the inner and the outer cylinders are $20 \mathrm{~mm}$ and $30 \mathrm{~mm}$, respectively, and the radius ratio $\eta$ is 0.667 . The inner cylinder rotates and the outer cylinder and the bottom wall are fixed. The working fluid is silicon oil and its kinematic viscosity, density and surface tension are $9.54 \times 10^{-5} \mathrm{~m}^{2} / \mathrm{s}, 935.0 \mathrm{~kg} / \mathrm{m}^{3}$ and $2.07 \times 10^{-3} \mathrm{~N} / \mathrm{m}$, respectively. A slit light source and a small quantity of aluminum flakes are used to visualize the flow in the meridional plane. 
The displacement of the free surface at the midpoint of the cylinders is measured by an ultrasonic sensor with a response time of $0.2 \mathrm{~ms}$ and a resolution of $0.2 \mathrm{~mm}$. When the lengths of the cylinders are infinite, the theoretical critical Reynolds number of the onset of the Taylor vortices is 76.4 [26].

The numerical simulation [23] is conducted under the condition of the experiment. The free surface is modelled by the VOF method. The governing equations are the unsteady axisymmetric Navier-Stokes equations, the equation of continuity and the conservation equation of the volume fraction $F$. The effects of the gravitational acceleration and the surface tension estimated from the curvature of the free surface profile are considered. The coordinate system is the cylindrical system with $(r, \theta, z)$ and the velocity components are $u, v$ and $w$. In the numerical formulation, physical quantities are made nondimensional by the reference length $d$ and the reference velocity $U$. Initially, the velocity components are zero in the entire flow region, and the inner cylinder is accelerated at the prescribed Reynolds number. In order to identify the critical Reynolds numbers of the transitions from the secondary modes to the primary modes, the secondary mode flow is decelerated in a quasi-steady state where the Reynolds number is reduced by $2 \%$ during the nondimensional time of 50 .

\section{RESULTS}

\subsection{Development process and spectrum of unsteady flow}

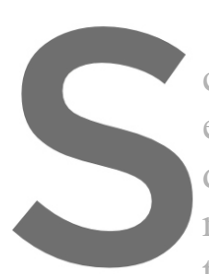

When the inner cylinder cylinder surface and they emerges usually after development process at $\Gamma=$ meridional plane at non the vortices rotating in the clockwise
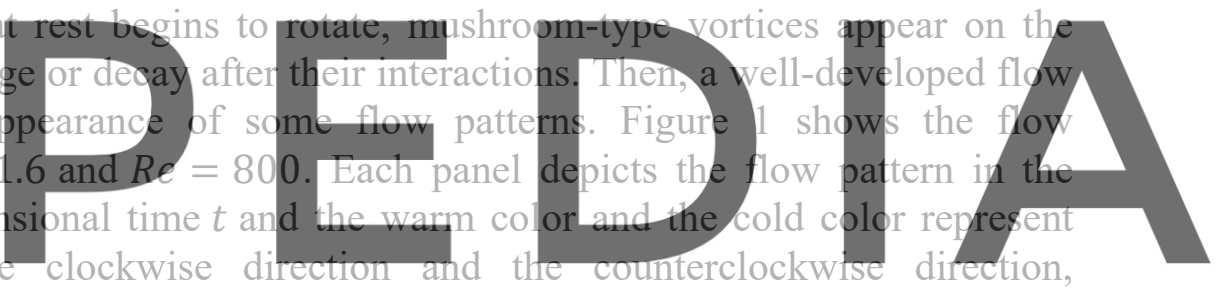
respectively. At $t=96.0$, three vortices exist in the meridional plane and the rotating

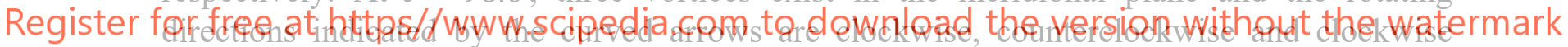
from the top to the bottom. This flow has radial outflow on the top and radial inflow on the bottom and the flow mode is the nomal 3-vontex mode $N 3$. This flow is transient. The upper two vortices decay and the final flow is N1 with one vortex. The well-developed flow at $\Gamma=$

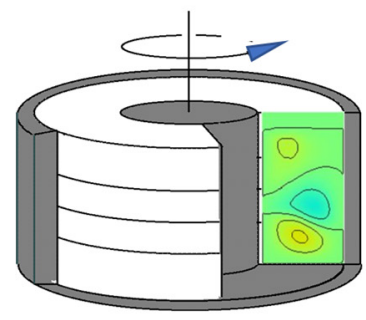

$t=64.0$
N3

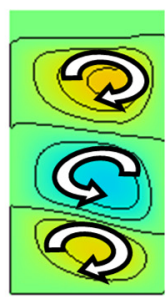

96.0

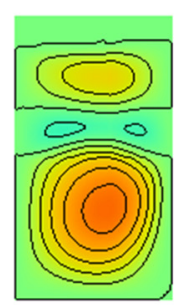

684.8

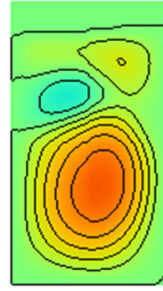

691.2
N1

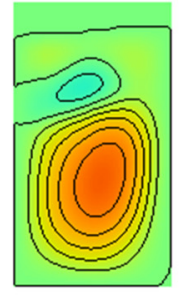

694.4

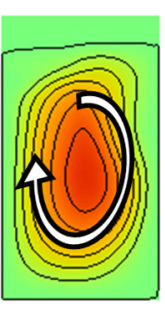

704.0

Figure 1 Development process of flow at $\Gamma=1.6, \operatorname{Re}=800$. 
2.2 and $R e=617$ presented in Figure 2 has two vortices. The lower vortex has a radial outflow on the bottom wall and it is anomalous, and the flow mode is the anomalous 2-vortex mode A2. This flow is found experimentally and numerically. Some details about the anomalous vortex on stationary walls were denoted by Watanabe et al. [21]. Figure 3 shows the flows at $\Gamma=2.4$. When the Reynolds number is 800 , the flow is the normal 3-vortex mode N3. Even in the same geometrical flow field, the flow at $R e=1000$ has an anomalous vortex on the bottom wall and the flow mode is A4.

The unsteady behavior of the free surface is one of the interesting phenomena in this kind of flows. Figure 4 represents the experimentally and numerically obtained power spectra of the displacement of the free surface and the numerically predicted power spectra of the

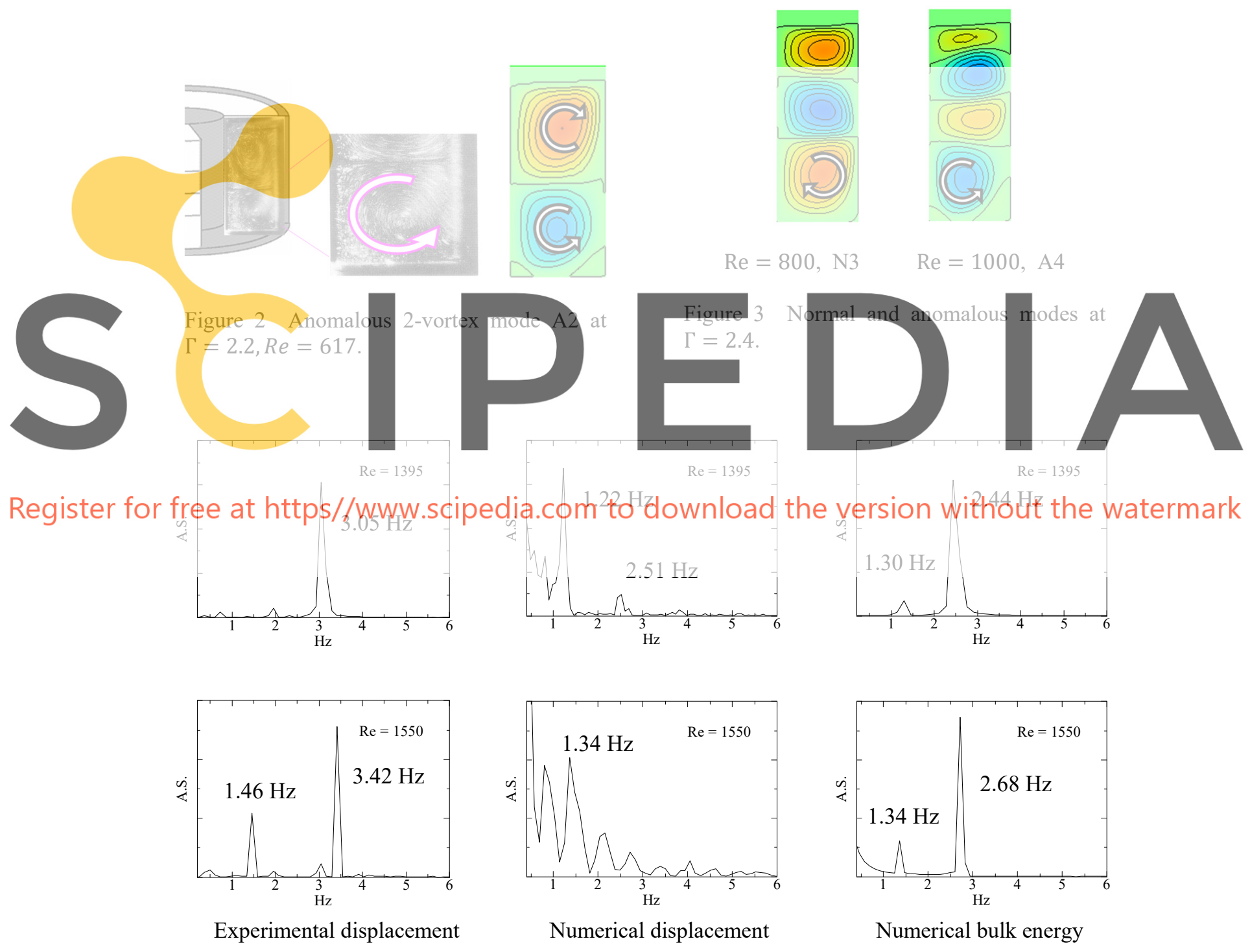

Figure 4 Power spectra of experimentally measured displacement, numerically predicted displacement and numerically predicted bulk energy. $\Gamma=5.6$. upper: $R e=1395$, lower: $R e=1550$. 
volume-averaged bulk kinetic energy. The numerical displacement is measured at the center point between two cylinders. The upper panels show the results at $R e=1395$ and the rotation frequency of the inner cylinder is $2.65 \mathrm{~Hz}$. The lower results are at $R e=1550$ and the rotation frequency is $2.94 \mathrm{~Hz}$. At the lower Reynolds number, the experimental result shows a simple harmonic motion at $3.05 \mathrm{~Hz}$. The numerical displacement has its first peak at $1.22 \mathrm{~Hz}$ and the higher peak at $2.51 \mathrm{~Hz}$, and these peaks do not match the experimental peaks. The spectrum of the energy has its first peak at $2.44 \mathrm{~Hz}$ and this almost corresponds to the peak in the experiment. When the Reynolds number is higher, the experimental displacement shows the first peak at $3.42 \mathrm{~Hz}$ and the second peak at $1.46 \mathrm{~Hz}$. The numerical displacement has some peaks and the first peak at $1.34 \mathrm{~Hz}$ approaches the second peak in the experiment. The first and the second peaks of the energy are $2.68 \mathrm{~Hz}$ and $1.34 \mathrm{~Hz}$, respectively, and the profile is similar to that of the experiment. The ultrasonic sensor capturing the displacement measures not a pin-point value but an averaged value in a finite area. This measurement in a finite area may be affected by the unsteady motion giving the kinetic energy.

\subsection{Flow mode transition}

As mentioned above, the secondary mode flows tend to appear at higher Reynolds numbers and the primary mode flows emerge at lower Reynolds numbers. In this section, we estimate the critical Reynolds numbers at which the secondary mode flows change to the primary mode flows when the flow is decelerated in quasi-steady states. The parameter space we

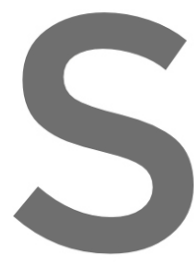
numerically study has the
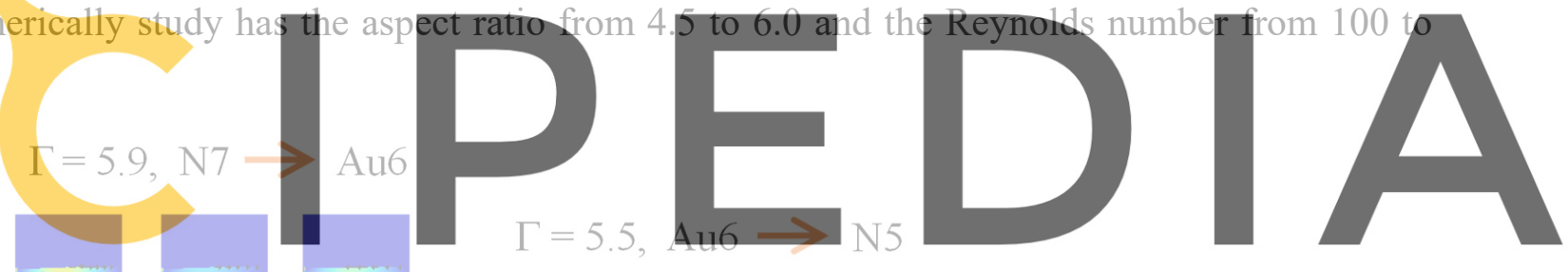

Register for free at https//www.scipedia.com
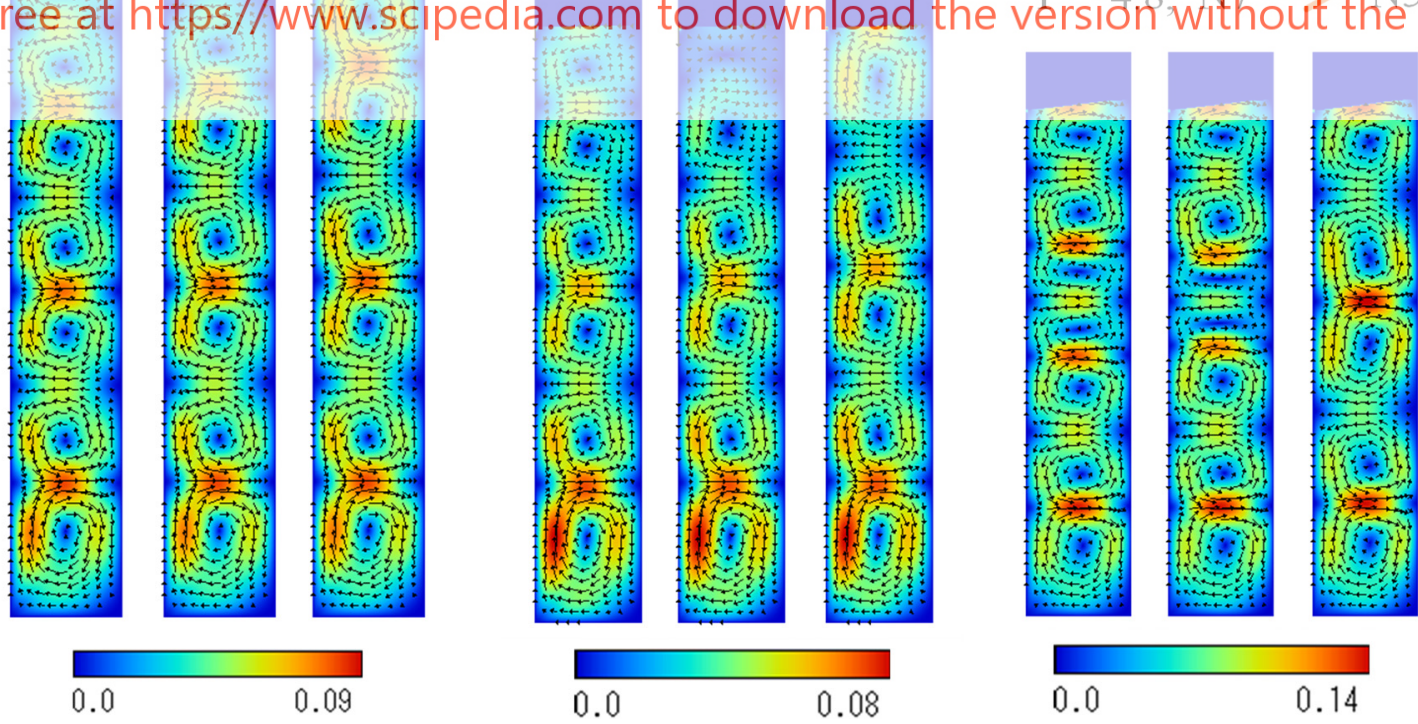

0.0

0.14

Figure 5 Flow pattern transitions in decelerating flows. 
500. The primary mode is N5 at $4.5 \leq \Gamma \leq 5.2$, and it is Au6 at $5.3 \leq \Gamma \leq 6.0$.

The transition processes of the decelerating vortex flows are shown as velocity vectors in the meridional sections in Figure 5. The color represents the value of the kinetic energy of the radial and axial velocity components. The left panel is the transition from N7 to Au6 at $\Gamma=$ 5.9. The change begins with the decay of the normal vortex at the top. The second vortex from the top grows and it collapses the top vortex, and the anomalous 6-vortex mode emerges with a radial inflow on the free surface (Au6). In the middle panel, the aspect ratio is 5.5 and the flow before the transition has a top anomalous vortex. With the deceleration, the top vortex weakens and the normal 5-vortex mode N5 forms. The right panel shows the transition from N7 to N5. The flow modes before and after the transition are normal, and not the top vortex but a pair of vortices in the flow field disappears. In this case, the third and fourth vortices become smaller and diminish. The aspect ratio of the case in the left panel is about 6.0 and the six-vortex mode is expected to be stable. The flow fields in the middle and right panels have the aspect ratio about 5.0 and the normal mode with five vortices is stable.

The experimental and numerical result of the bifurcation diagram of the primary and the secondary modes are represented in Figure 6. When the aspect ratio is 6.0, the experimental and numerical results show the critical Reynolds number of the transition from N7 to Au6 is about 100. The critical Reynolds number of the transition from N7 to N5 is about 200 at $\Gamma=$ 5.0. However, the overlapping range of the aspect ratio of the experimental and numerical results is limited. This is because of the difficulty to find the transition experimentally and

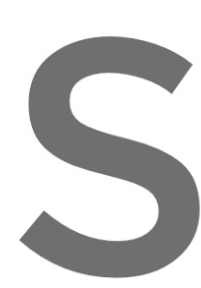
numerically. The results of the transition experiment, the critica increases from 5.5 to critical Reynolds numb experimental condition experimental setup.
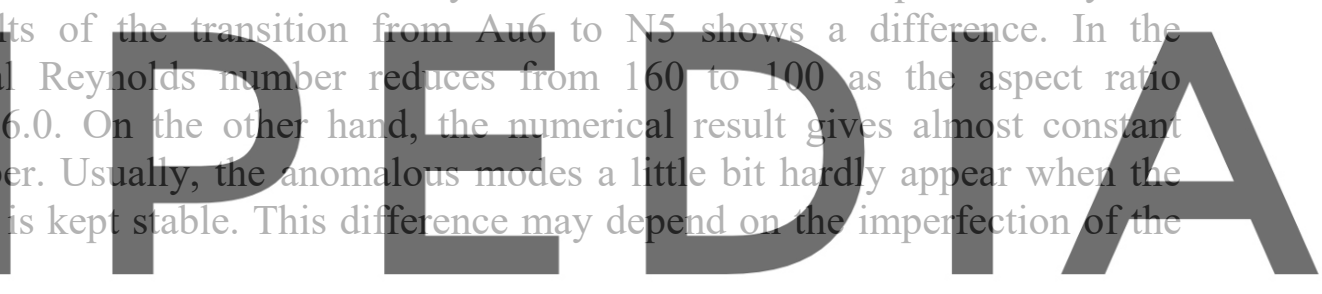

Register for free at https//www.scipedia.com to download the version without the watermark
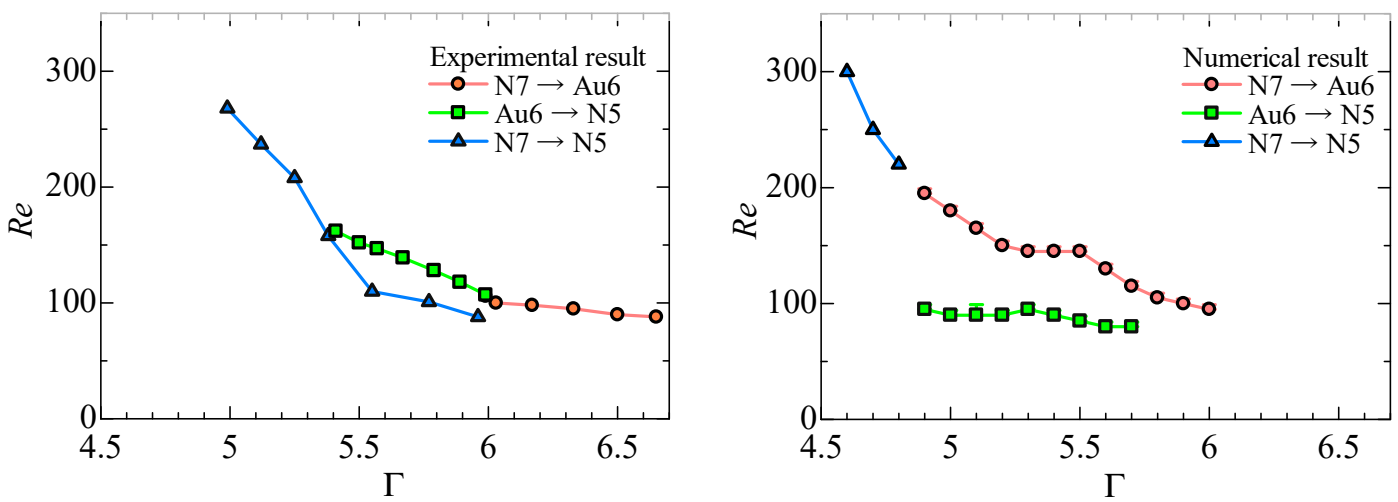

Figure 6 Bifurcation diagram between the primary mode and the secondary mode. 


\section{CONCLUSIONS}

- Numerical and experimental study was carried out on the vertical Taylor-Couette system with a free surface at the top. The inner cylinder rotates and the outer cylinder and the bottom wall are stationary. The cylinders are long enough to include the free surface in the annulus of the cylinders. In the experiment, the flow patterns in the meridional section are observed and the displacement of the free surface is measured. The numerical approach is used to predict the unsteady flow development. As has been known, some flow modes appear in a fixed geometrical configuration, which depend on the Reynolds number.

- Though the numerically obtained power spectra of the displacement of the free surface includes its peaks corresponding those in the experimental result, the numerically estimated kinetic energy spectra shows a reasonable agreement with the spectra of the experimentally measured displacement.

The anomalous modes with a radial outflow on the bottom wall and/or a radial inflow at the top free surface are identified.

The numerically predicted transition from N7 (normal 7-vortex mode) to Au6 (anomalous 6-vortex mode with an upper anomalous vortex) and the one from N7 to N5 (normal 5-vortex mode) have favorable agreements with the experimental results. However, the numerical and experimental results show a difference in the transitions from Au6 to N5.
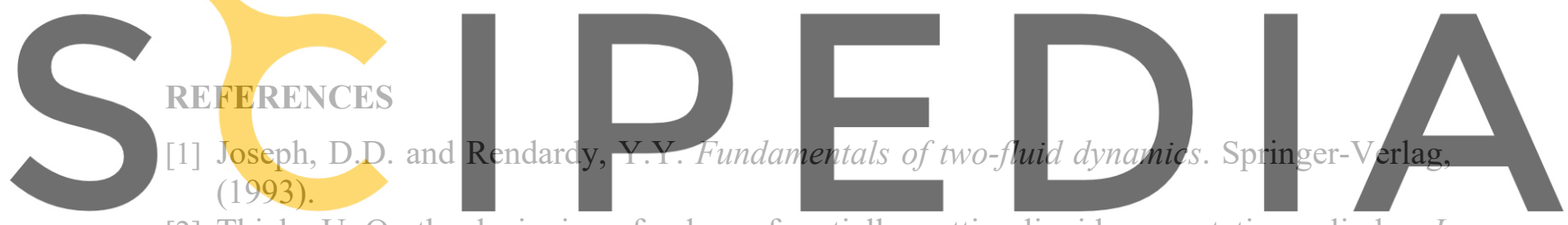

[2] Thiele, U. On the depinning of a drop of partially wetting liquid on a rotating cylinder. $J$.

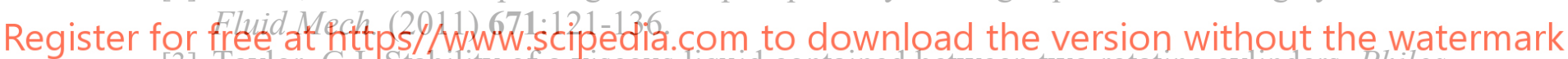

[3] Taylor, G.1. Stability of a viscous liquid contained between two rotating cylinders. Philos. Trans. R. Soc. London A (1923) 223:289-343.

[4] Grossmann, S., Lohse, D. and Sun C. High-Reynolds number Taylor-Couette turbulence. Annu. Rev. Fluid Mech. (2016) 48:53-80.

[5] Hubacz, R. and Wronski, S. Horizontal Couette-Taylor flow in a two-phase gas-liquid system: Flow patterns. Exper. Thermal Fluid Sci. (2004) 28:457-466.

[6] Dluska, E., Wronski, S. and Ryszczuk, T. Interfacial area in gas-liquid Couette-Taylor flow reactor. Exper. Thermal Fluid Sci. (2004) 28:467-472.

[7] Peixinho, J., Mirbod, P., and Morris, J.F. Free surface flow between two horizontal concentric cylinders, Euro. Phys. J. E (2012) 35:19-1-9.

[8] Baier, G. and Graham, M.D. Two-fluid Taylor-Couette flow with countercurrent axial flow: linear theory for immiscible liquids between corotating cylinders, Phys. Fluids (2000) 12:294-303.

[9] Zhu, X. and Vigil, R.D. Banded liquid-liquid Taylor-Couette-Poiseuille flow, AIChE J. (2001) 47:1932-1940.

[10] Nakase, M., Makabe, R. and Takeshita, K. Relation between oil-water and extraction performance in liquid-liquid countercurrent centrifugal extractors with Taylor vortices. $J$. 
Nucl. Sci. Tech. (2013) 50:287-295.

[11] Sathe, M.J., Deshmukh, S.S., Joshi, J.B. and Koganti, B. Computational fluid dynamics simulation and experimental investigation: study of two-phase liquid-liquid flow in a vertical Taylor-Couette contactor. Ind. Eng. Chem. Res. (2010) 49:14-28.

[12] Broadbent, A.L., Mullin, J.M., Codd, S.L., Dockery, J.D. and Seymour, J.D. Pulsed gradient spin echo nuclear magnetic resonance measurement and simulation of two-fluid Taylor vortex flow in a vertically oriented Taylor-Couette device. Appl. Magn. Reson. (2012) 42:134-152.

[13] Sugiyama, K. Calzavarini, E. and Lohse, D. Microbubbly drag reduction in TaylorCouette flow in the wavy vortex regime, J. Fluid Mech. (2008) 608:21-41.

[14] Gao, X., Kong, B. and Vigil, R.D. CFD investigation of bubble effects on TaylorCouette flow patterns in the weakly turbulent vortex regime. Chem. Engng. J. (2015) 270:508-518.

[15] Linek, M. and Ahlers, G. Boundary limitation of wave numbers in Taylor-vortex flow. Phys. Rev.E (1998) 58:3168-3174.

[16] Deng, R., Arifin, D.Y., Mak Y.C. and Wang C.-H. Characterization of Taylor vortex flow in a short liquid column. AIChE J. (2009) 55:3056-3065.

[17] Mulligan, S., Cesare G.D., Casserly, J. and Sherlock, R. Understanding turbulent freesurface vortex flows using a Taylor-Couette flow analogy. Scientific Report (2018) 8:824$1-14$.

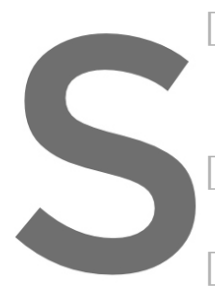

[18] Larcher, T.,

Instabilities and

rotating annulus.

Benjamin, T.

experiment. J. Fluid Mech

Nakamura, I.
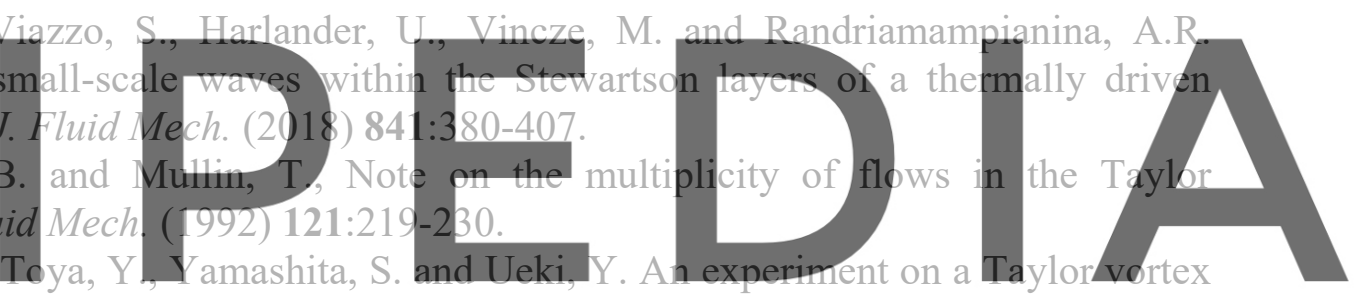

flow in a gap with a small aspect ratio (Bifurcation of flows in a symmetric system).

Register for frede a

[21] Watanabe, T., Furukawa, H. and Nakamura, I. Nonlinear development of flow patterns

in an annulus with decelerating inner cylinder. Phys. Fluids (2002) 14:333-341.

[22] Toya, Y., Nakamura, I., Yamashita, S. and Ueki, Y. An experiment on a Taylor vortex flow in a gap with a small aspect ratio. Bifurcation of flows in an asymmetric system. Acta Mechanica (1994) 102:137-148.

[23] Watanabe, T. and Toya, Y. Vertical Taylor-Couette flow with free surface at small aspect ratio. Acta Mechanica (2012) 223:347-353.

[24] Adnane, E., Lalaua, A. and Bouabdallah, A. Experimental study of the laminarturbulent transition in tilted Taylor-Couette system subjected to free surface effect. J. App. Fluid Mech. (2016) 9:1097-1104.

[25] $\mathrm{Xu}, \mathrm{W}$. and Harlander, U. Inertial mode interactions in a rotating tilted cylinder annulus with free surface. Phys. Rev. Fluids (2020) 5:094801-1-21.

[26] Koschmieder, E.L. Bénard cells and Taylor vortices. Cambridge Univ. Press, (1993). 